\title{
Evidence for Health-Promoting Properties of Lepidium sativum L.: An Updated Comprehensive Review
}

\author{
(1) Yalda HEKMATSHOAR ${ }^{1,2}$, (1) Tülin ÖZKAN1,3, (1) Yalda RAHBAR SAADAT4* \\ 1 University of Missouri, School of Medicine, Department of Child Health, Columbia, USA \\ ${ }^{2}$ Altınbaș University, School of Medicine, Medical Biology Department, İstanbul, Türkiye \\ ${ }^{3}$ Ankara University, Faculty of Medicine, Department of Medical Biology, Ankara, Türkiye \\ ${ }^{4}$ Tabriz University of Medical Sciences, Kidney Research Center, Tabriz, Iran
}

\begin{abstract}
Lepidium sativum $\mathrm{L}$. is a common herb distributed worldwide, used as a food ingredient and therapeutic agent in traditional medicine for treating health-related disorders. L. sativum and its extracts have been described to possess numerous biological activities including antimicrobial, antidiabetic, antioxidant, antidiarrheal, anticancer, and numerous health-promoting effects in in vivo and in vitro studies. The purpose of this review is to summarize the findings describing important biological functions and therapeutic effects of $L$. sativum in various cell lines and animal models. In this review, the English-language articles were gathered from electronic databases including Web of Science, PubMed and Google Scholar with no time limit applied to any database. The search terms used in this review include, "Lepidium sativum L." and/or "chemical composition", "health benefits", “antimicrobial", “antioxidant", “anticancer", “diuretic", “nephro-protection", "antidiarrheal", “antidiabetic", “anti-asthmatic", "neuroprotection", "metabolic", "bone fracture", and "reproductive performance". Additional and eligible studies were collected from reference lists of appropriate articles. The information presented will be helpful to attract more interest toward medicinal plants by defining and developing novel clinical applications and new drug formulations in the future. Pre-clinical studies showed that $L$. sativum possesses potent health-promoting effects involving various molecular mechanisms. Taken all together, data suggested that identified herbal plants such as $L$. sativum, can be exploited as nutritional and therapeutic agents to combat various ailments. Despite much research in this field, further comprehensive in vitro/in vivo studies and clinical trials are needed to identify the mechanisms underlying the biological and therapeutic activities of $L$. sativum.
\end{abstract}

Key words: Ethnomedicine, medicinal plants, Lepidium sativum, nutraceutical, therapeutic agents

\section{INTRODUCTION}

Many plants have been considered a principal source of potent therapeutic drugs for centuries. Lepidium sativum L., (alias Garden cress) is a fast-growing perennial herb with edible leaves that grows up to $50 \mathrm{~cm}$ in height and belongs to the family Brassicaceae (Cruciferae).-1 It is widely dispersed throughout the world; Africa, Asia, Australasia, Europe, Northern, and Southern America. ${ }^{4-6}$ The leaves and seed oils are commonly applied in traditional medicine to treat various clinical complications, including asthma, hypertension, hyperglycemia, hepatitis, menstrual problems, sexual debility, arthritis, fracture, diarrhea, vitamin C deficiency, constipation, and migraine..$^{2,7,8}$ Additionally, they have been represented with pharmacological properties such as immunity booster, anticancer, antioxidant, laxative, febrifuge, diuretic, and galactogogue activities (Figure 1)..$^{1.9-11}$

The chemical composition of the L. sativum seeds illustrated that they contain high levels of proteins, fatty acids (oleic and linolenic acids), crude fiber (lignans, etc.), essential minerals (potassium, phosphorus, calcium, and iron), phytosterols (sitosterol, campesterol, and avenasterol), carotenoids, alkaloids (lepidine, $N, N^{\prime}$-dibenzylthiourea, $N, N^{\prime}$-dibenzyl urea, 


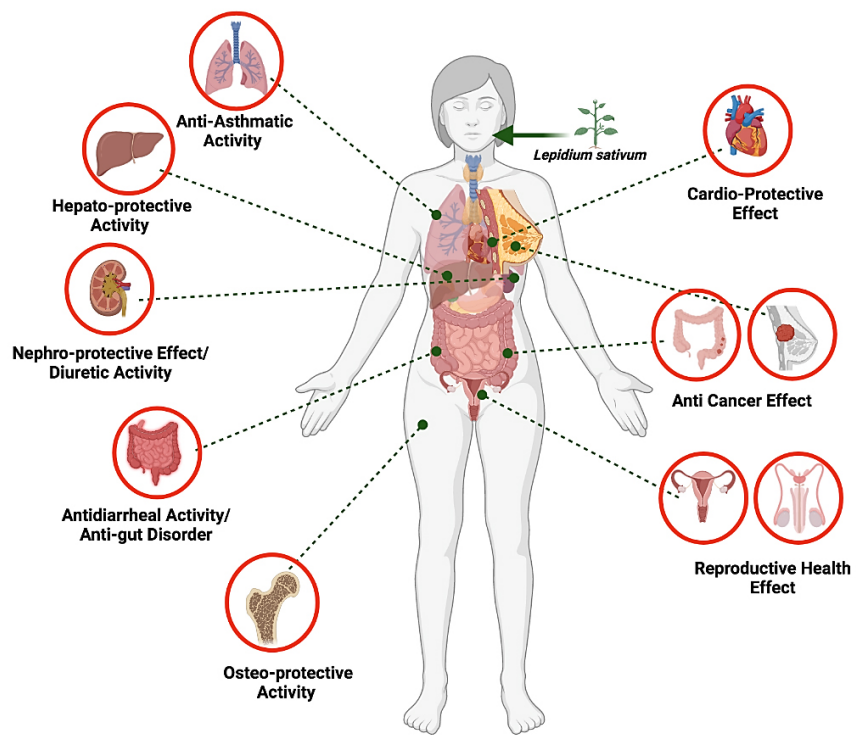

Figure 1. Therapeutic effects of Lepidium sativum under various physiological/pathological conditions

sinapine), hydroxycinnamic acids (sinapic acid), ${ }^{12}$ glucosinolates (glucotropaeolin and 2-phenyl ethyl glucosinolates) ${ }^{13}$ riboflavin, ascorbic acid, and tocopherols.,2,14-16

Toxicological studies state that $L$. sativum seeds are considered practically non-toxic and safe. ${ }^{17}$ Nowadays, ethnomedicinal studies gained great attention due to their beneficial roles against various ailments, though proper identification and documentation of medicinal plants seems indispensable.18,19 Here, we provided an updated comprehensive overview of the chemical composition of $L$. sativum with focusing on its beneficial impacts, medicinal utility, and underlying mechanisms.

\section{Chemical composition of L. sativum}

Various factors (including variety, plant agronomic practices, seed collection stage, and geological conditions) contribute to the differences of the chemical composition of $L$. sativum seeds. ${ }^{20}$ L. sativum seeds consist of carbohydrates, protein, lipids, and fiber. ${ }^{21}$ L. sativum seeds also contain mucilaginous substances (cellulose and uronic acid containing polysaccharides). Additionally, the seeds have high protein and lipid contents, minerals as well as vitamins (i.e. vitamins A, C, D, B6, and cobalamin). ${ }^{22}$ The presence of numerous components has been reported in L. sativum seeds including alkaloids, phenolic compounds, anthraquinones, and cardiac glycosides, flavonoids, tannins, benzoic, dihydroxybenzoic, gallic, chlorogenic, 4-hydroxycoumaric, vanillic, and salicylic acids, pyrogallol, catechin, caffeine, isoleucine as well as different imidazole alkaloids e.g. lepidine and semilepidine. ${ }^{20}$ Essential (leucine, valine, lysine, phenyl alanine, isoleucine, arginine, histidine, threonine, and methionine) and non-essential (glutamic acid, aspartic acid, glycine, proline, serine, alanine, and tyrosine) amino acids are present in L. sativum seeds. Most abundant fatty acid in L. sativum seeds is $\alpha$-linolenic acid (ALA), however, oleic, palmitic, stearic, arachidic, linoleic, lignoceric, behenic, acids $\beta$-sitosterol are found in different concentrations. L. sativum seed oils comprise high amounts of $\gamma$-tocopherol as well as $\alpha$-tocopherol. Benzylcyanide and benzyl isothiocyanate are detected as significant volatile components of the seeds. ${ }^{20,23}$ In a recent research, the results of the liquid chromatography-mass spectroscopy (LC-MS) of L. sativum extracts demonstrated various secondary metabolites (including kaempferol, apigenin, luteolin, quercetin, and 7-hydroxy-4',5,6trimethoxyisoflavone, chlorogenic acid, sinapic acid, ascorbic acid, $p$-coumaric acid, 6-prenylnaringenin, and $\alpha$-tocopherol) in different concentrations. ${ }^{24}$

\section{Beneficial effects of $L$. sativum}

\section{Antimicrobial activity}

The growth of antibiotic resistance in bacterial strains and adverse effects of synthetic antibiotics provide a route to exploiting plants with strong medical potential in treating bacterial infections. $^{2}$ Many studies have reported that the L. sativum extract is effective against bacterial strains and pathogens. It has been proposed that the antibacterial potency of $L$. sativum depends on benzyl isothiocyanate presence. ${ }^{25}$ An increasing number of studies confirmed L. sativum's antimicrobial properties (Table 1), however, exact mechanism of action, which elucidates how they could perform such activities has not fully understood. Recently, Al-Otaibi et al. ${ }^{26}$ revealed probable therapeutic potential of the methanolic extract of $L$. sativum seeds in Trypanosoma evansi (a parasitic protozoan) infected-Swiss albino mice. Their findings showed that the methanolic extract treatment results in restoring the hematology analysis (haemoglobin content, hematocrit, erythrocyte count, leucocyte count, and percentage of lymphocytes) to the preinfection values. Besides, the study discovered that the intraperitoneal (ip) injection of the extract exerts more efficacy rather than oral administration. ${ }^{26}$ In another research it has been shown that the leaf extract of $L$. sativum could inhibit the viability of the protoscolices Echinococcus granulosus. ${ }^{27}$

Al-Marzoqi et al. ${ }^{28}$ examined the antimicrobial potential of the crude alkaloid, phenolic, and terpenoid compounds of $L$. sativum extract. Their findings demonstrated that both Grampositive and Gram-negative pathogens (Staphylococcus aureus, S. epidermedis, S. saprophyticus, Klebsiella, Serratia, Proteus, Escherichia coli, Pseudomonas, and Provedenatia) were resistant to phenolic compounds, whereas the alkaloid and terpenoid compounds exerted an extensive antimicrobial activity against Gram-positive and Gram-negative bacteria. Over all, they suggested that the hydrophobicity of components of the plant extracts, leads to disruption of bacterial cell membrane lipids and mitochondria that in turn causes microbial death. ${ }^{28}$ They also showed that different concentrations of active components of the aforementioned plant demonstrated diverse effects on various pathogenic organisms. ${ }^{25}$ 
Table 1. Antimicrobial activity of various extracts of Lepidium sativum

\begin{tabular}{|c|c|c|}
\hline Lepidium sativum & Antimicrobial activity & References \\
\hline $\begin{array}{l}\text { Seed petroleum ether, methanol, and } \\
\text { water extracts }\end{array}$ & $\begin{array}{l}\text { Escherichia coli, Staphylococcus aureus, Klebsiella pneumonae, Proteus vulgaris, } \\
\text { Pseudomonas aeruginosa, and Candida albicans }\end{array}$ & 2 \\
\hline Seed extract & K. pneumonia, E. coli, S. aureus, and Bacillus cereus & 7 \\
\hline Seed extract & S. aureus, B. subtilis, E. coli, $P$. aeruginosa, S. enterica, K. pneumoniae, and $C$. albicans & 29 \\
\hline Chloroform, methanol extract & $\begin{array}{l}\text { E. coli, S. typhi, S. aureus, B. subtillis, Aspargillus niger, Fusarium oxysporum, and } \\
\text { Fusarium solani }\end{array}$ & 30 \\
\hline $\begin{array}{l}\text { Ethanol, methanol and chloroform } \\
\text { extracts }\end{array}$ & E. coli, K. pneumoniae, P. aeruginosa, S. aureus, and Shigella sonnie & 31 \\
\hline $\begin{array}{l}\text { Seed methanol, and ethyl acetate } \\
\text { extracts }\end{array}$ & Rhodococcus equi & 32 \\
\hline $\begin{array}{l}\text { Whole plant water, methanol and ethanol } \\
\text { extracts }\end{array}$ & Multi-drug resistant E. coli & 33 \\
\hline Crude extract of seeds & $\begin{array}{l}\text { P. aeruginosa, E. coli, S. aureus, B. subtilis, and two fungi species (Aspergillus niger } \\
\text { and C. albicans) }\end{array}$ & 34 \\
\hline
\end{tabular}

\section{Antioxidant activity}

Formation of reactive oxygen species (ROS) is provoked through normal metabolism, however, excessive amounts are detrimental and should be scavenged to avoid any damage. ${ }^{35}$ Oxidative stress is implicated in the pathogenesis of several chronic ailments such as cancer, cardiovascular disease, and etc. ${ }^{36}$ Intake of nutraceuticals (rich in antioxidants) from different herbs possibly protect human body against free radicals, thus, alleviate oxidative damage and degenerative diseases. ${ }^{36}$ In a study conducted by Aydemir and Becerik ${ }^{36}$, L. sativum seed extract exhibited antioxidant activity. In another study, antioxidant activity of $L$. sativum seed oil (petroleum ether) was evaluated employing free radical (2,2-diphenyl-1-picrylhydrazyl, DPPH) scavenging activity method. The petroleum ether extract exerted antioxidant activity dose-dependently. ${ }^{29}$ Omer et al. ${ }^{34}$ reported that the ethyl acetate fractions of the $L$. sativium seeds had highest antioxidant activity. Another study conducted by Malar et al. ${ }^{37}$ revealed the substantial antioxidant activity of the ethanolic extract of $L$. sativum plant parts (shoot, leaf, stem, and seed). Further, it has been shown that the presence of flavonoid and tannin in the methanolic extract of $L$. sativum leads to the significant antioxidant activity. ${ }^{32}$

The cyanobacterial toxins and crude extract provoke oxidative stress response in $L$. sativum seedlings through lipid peroxidation, elevation of the levels of tocopherol and antioxidant enzymes (including glutathione peroxidase, glutathione S-transferase, and glutathione reductase). ${ }^{35}$ Furthermore, in a study performed by Kasabe et al. ${ }^{38}$ it has been elucidated that the seeds of $L$. sativum possess antioxidant activities due to total phenolic content of the seeds. In a recent study, it was reported that methanolic extract of $L$. sativum exerted potent radical scavenging activity comparing to the ethanolic extract. ${ }^{23}$

\section{Anticancer activity}

Cancer has remained as the leading cause of death worldwide. Nowadays, applying natural remedies to overcome the side effects of conventional methods in cancer treatment have received growing attention. ${ }^{39,40}$ L. sativum demonstrated anticancer, antiproliferative and cytotoxic effects through different mechanisms such as induction of apoptosis and necrosis in various cancer cells. An in vitro study carried out on breast cancer cell line (MCF-7), reported the apoptosis induction capability of the aqueous extract of $L$. sativum seeds. However, high concentrations of the extract resulted in necrosis. ${ }^{41}$ Recently, El Sayed et al. ${ }^{42}$ reported the antioxidant and anti-mutagenic effects of $L$. sativum against in vivo Ehrlich ascites carcinoma (EAC) in Swiss albino mice. Their findings demonstrated anticancer effect of $L$. sativum in EAC tumor-bearing mice lifespan. Additionally, increased levels of liver enzymes and glutathione peroxidase activity as well as decreased levels of malondialdehyde (MDA) were observed, which in turn indicated the antioxidant properties of the extract. Besides, L. sativum extract decreased chromosomal aberration and DNA fragmentation. ${ }^{42}$ In another study conducted by Selek et al. $^{43}$, L. sativum methanolic extract substantially induced apoptosis in human peripheral lymphocyte cells, colon cancer (DLD-1), and endometrium cancer cell lines (ECC-1) in a dosedependent pattern, besides, the extract presented significant antioxidant activity. Taken all together, they suggested that high levels of phenolic and flavonoid compounds of the extract may be considered as the underlying mechanism for the anticancer activity of $L$. sativum. ${ }^{43}$ The antiproliferative effects of the leaf aqueous extracts of $L$. sativum were explored on human tongue squamous carcinoma (CAL-27). The aqueous extract inhibited growth of CAL-27 cells concentration-dependently. Apoptosis induction and DNA damage was observed in L. sativum extracttreated cancer cells. ROS generation in the mitochondria of the treated cells seems the cause of apoptosis induction. ${ }^{44}$ According to a recent study, the hydroalcoholic extract of $L$. sativum showed cytotoxicity on HeLa cell line. ${ }^{45}$ Further, Aslani et al. ${ }^{46}$ evaluated cytotoxic effects of hydro-alcoholic extracts of $L$. sativum shoots in $K 562$ cell line as a model of CML. MTT assay results depicted that the extract exerted cytotoxic effect on $\mathrm{K} 562$ cell line in a dose and time dependently. ${ }^{46}$ 


\section{Effects on urinary system}

\section{Diuretic activity}

There are different studies suggested that the plant's diuretic effect may depend on presence of phytochemicals such as flavonoids, saponins, steroids or organic acids. ${ }^{47}$ In line with this, Patel et al. ${ }^{47}$ illustrated that the aqueous and methanolic extracts of $L$. sativum dose-dependently augmented urine secretion in rat models. They suggested that $L$. sativum extracts' diuretic activity possibly induced by individual or synergistic effects of flavonoids and steroids, which in turn leads to increased local blood flow and vasodilation or inhibition of water and anions tubular reabsorption. ${ }^{47}$ In addition to excessive urine production, increased sodium and water excretion contributes to L. sativum's antihypertensive effect. ${ }^{47}$ Maghrani et al. ${ }^{48}$ investigated diuretic and antihypertensive properties of the aqueous extract of $L$. sativum in normotensive and spontaneously hypertensive rats (SHR). Oral administration of the extract caused substantial drop in blood pressure as well as increase of urinary exertion of sodium, potassium, and chlorides in SHR rats. ${ }^{48}$

\section{Nephroprotective effect}

Numerous evidence elucidates some medications have potential to induce nephrotoxicity and acute renal failure which causes loss of renal functions. Phytochemicals of $L$. sativum may have antioxidant activity, which, thus, overcome the drug-induced nephrotoxicity. ${ }^{49}$ In this regard, Yadav et al. ${ }^{49}$ depicted that the ethanolic extract of $L$. sativum exerts nephroprotective and curative activity against cisplatininduced nephrotoxicity in Wistar rats. The administration of the extract markedly declined the levels of urea, creatinine as well as lipid peroxidation and enhanced glutathione (GSH) levels. ${ }^{49}$ Recently, it has been presented that $L$. sativum seed aquatic extract could ameliorate oxidative stress induced by dexamethasone in rats. Dexamethasone administration led to the elevation of thiobarbituric acid reactive substances (TBARS), hydrogen peroxide, liver function biomarker levels, and lactate dehydrogenase (LDH) activity. However, enzymatic and non-enzymatic antioxidants, protein content, and alkaline phosphatase (ALP) activity were markedly reduced. The aquatic extract administration in rats received dexamethasone, could alleviate lipid peroxidation, antioxidant status, and biochemical indices, when compared to the dexamethasone-treated group. ${ }^{50}$ Furthermore, administration of $L$. sativum powder to the gentamicin-induced nephropathy in diabetic albino rats caused a substantial reduction of the serum levels of glucose, MDA, augmentation of the glutathione transferase (GST), superoxide dismutase (SOD), total antioxidant capacity (TAC), glutathione pyroxidase (GPX), and catalase (CAT) activity as well as serum insulin levels, though exerted nephoprotective effect by enhancing renal damage..$^{51}$

\section{Effects on digestive tract}

\section{Antidiarrheal activity}

A few studies reported the antidiarrheal and antispasmodic properties of $L$. sativum. ${ }^{9,52}$ In a study performed in rat model, the administration of extract can inhibit castor oil-induced diarrhea like dicyclomine. Data from the study proposed that dual suppression of muscarinic receptors and $\mathrm{Ca}^{2+}$ channels was responsible for the antidiarrheal/antispasmodic activities of the L. sativum. Moreover, presence of gut relaxant compounds and phytochemicals such as alkaloids, and $\beta$-sitosterol, plays an important role in L. sativum antidiarrheal/antispasmodic effect. ${ }^{9}$ Additionally, they examined antidiarrheal/antispasmodic properties of the crude extract of $L$. sativum seeds in multiple species (mice, Sprague-Dawley rats, guinea-pigs, and local breed rabbits). They also depicted the antidiarrheal/ antispasmodic mechanisms specific to each species as below: 1) in rabbit model: activation of $\mathrm{K}^{+}$channels and blockade of PDE enzyme, 2) in guinea-pig model: anti-muscarinic and weak $\mathrm{Ca}^{2+}$ antagonist-like pathways and 3 ) in rat model: a combination of anti-muscarinic, $\mathrm{Ca}^{2+}$ antagonist and PDE-inhibitory-like mechanisms. ${ }^{53}$

\section{Effect on gut disorders}

The aqueous-methanolic extract of $L$. sativum seeds were reported to be potent contributors to indigestion and constipation (as digestive disorders). Najeeb-Ur-Rehman et al. ${ }^{54}$ described an in vivo experiment conducted in mouse model displaying the atropine-sensitive pro-kinetic and laxative properties, which were relatively mediated through muscarinic receptors.

\section{Metabolic activity}

Findings of a recent study depicted that ethanolic and aqueous extracts of L. sativum significantly exerted hepato-protective, hypolipidemic, hypoglycemic, hypoinsulinemic, anti-obesity, antioxidant, and anti-inflammatory properties in high fat dietfed rats. Moreover, the hepatic tissues of ethanolic/aqueous extracts-treated rats demonstrated upregulation of the intracellular phosphorylation of common markers of insulin signaling cascade ( $p$-IR/p-AKT/pmTOR/p-p70S6K). Both extracts mitigated lipid peroxidation and restored the amounts of antioxidant enzymes. ${ }^{55} \mathrm{Al}$-Asmari et al. ${ }^{56}$ designed a study to assess the hepatoprotective effect of ethanolic extract of $L$. sativum against carbon tetrachloride $\left(\mathrm{CCl}_{4}\right)$-induced toxicity in rat model. Their findings demonstrated that the level of serum alanine transaminase (ALT), ALP, aspartate transaminase (AST), and bilirubin was significantly decreased in ethanolic extract-treated rats. Additionally, histological analysis of liver tissues exhibited mild necrosis and inflammation in extracttreated group in comparison to the $\mathrm{CCl}_{4}$-treated group. ${ }^{56}$

Similar findings were obtained by Zamzami et al. ${ }^{57}$ for hepatoprotective effects of $L$. sativum seeds against $\mathrm{CCl}_{4}-$ induced hepatic injury in New Zealand rabbits. The extracttreated rabbits showed significant reduction in serum levels of liver biomarkers (transaminases, $\gamma$-GT, and ALP), total bilirubin, cholesterol, triglycerides (TG) and elevated levels of total protein and albumin. Moreover, L. sativum extract reduced oxidative stress in liver tissues. Overall, biochemical analysis as well as histopathological examination revealed that L. sativum extract effectively could reverse the hepatotoxicity of $\mathrm{CCl}_{4}$ in vivo. ${ }^{57}$ In another study, potential protective and therapeutic effects of $L$. sativum against aluminum-induced injury of liver and kidney in albino rat were investigated. Data 
from this experiment exhibited that administration of the extract led to a marked reduction in levels of serum biomarkers of liver (e.g. AST, ALT, ALP, bilirubin, urea, and creatinine) and kidney functions. It is also significantly augmented total protein and albumin. Besides, rats fed with the extract reversed necrosis of hepatocytes, glomeruli, and renal tubules. It has been suggested that the antioxidant properties of $L$. sativum seeds exerted the aforementioned beneficial effects. ${ }^{58}$

L. sativum seed powder exhibited the potent cardioprotective effect against 5-fluorourasil (FU)-induced cardiotoxicity and oxidative stress in albino rats. L. sativum seed powder significantly reduced the inflammatory markers [myocardial IL-1 $\beta$ and myeloperoxidase (MPO) activity], concentration of serum cardiac biomarkers (CK-MB and CTnl), whereas it increased gluthathion (GSH) concentration. Moreover, in the $L$. sativum-treated group, the hypertriglyceridemia and hypercholesterolemia factors were returned to the normal status compared to the 5-FU-induced cardiotoxicity group. ${ }^{59}$ In another research, hypolipidemic activity of $L$. sativum seed extract against triton $x-100$ and high cholesterol diet (HCD)induced hyperlipidemia was investigated on rats. Their results showed that the extracts significantly protected against all parameters [total cholesterol (TC), TG, low density lipoprotein cholesterol (LDLC), very low density lipoprotein cholesterol (VLDLC)] of HCD diet-induced hyperlipidemia, thus, may exert anti-hyperlipidemic effect. ${ }^{60}$ Additionally, Raish et al. ${ }^{61}$ evaluated hepatoprotective effect of $L$. sativum ethanolic extract in rat model with liver damage induced by D-galactosamine/ lipopolysaccharide. Data from their study revealed that the extract significantly down-regulated the pro-inflammatory cytokines (e.g. TNF $\alpha$ and IL-6 mRNA), stress genes (iNOS and $\mathrm{HO}-1)$ and up-regulated the $\mathrm{IL}-10$ expression dosedependently. Furthermore, the extract pretreatment leads to down-regulation of nuclear NF-KB (p65), NF-kB-DNA binding activity, MPO activity, and nitric oxide (NO) level. Additionally, it can down-regulate caspase 3 and up-regulate $\mathrm{Bcl}-2$ protein expression, which overall indicated that $L$. sativum markedly alleviates hepatic damage through reduction of oxidative stress, inflammation, and apoptosis in the liver. ${ }^{61}$ Administration of $L$. sativum seed ethanolic extract effectively could ameliorate triacylglycerol, LDLC, and TC as well as downregulation of hepatic 3-hydroxy-3-methylglutaryl-coenzyme A reductase and VEGF expression in rat NAFLD model, thus, in turn, impedes obesity, NAFLD, NASH, and fibrosis. Additionally, the extract administration exerted antioxidant activity via increasing GSH, SOD, and CAT activities as well as reduction of MDA and NO levels. ${ }^{62}$

In another study, Sakran et al..$^{63}$ isolated 5,6-dimethoxy-2', $3^{\prime}$ methylenedioxy-7-C- $\beta$-D-gluco-pyranosyl isoflavone (a new isoflavonoid) from $L$. sativum seeds. They showed that this new isoflavonoid have a potential to diminish the hepatotoxicity induced by paracetamol in adult Sprague Dawley male rats. They proposed that hepatoprotective effect depends on enhancing TAC and normalizing level of liver enzymes including GSH, SOD, GPX, CAT, and GST.63

\section{Antidiabetic activity}

Hyperglycemia (high blood sugar) causes long-term complications in affected people. Untreated hyperglycemia results in renal failure, diabetic cataract, elevated risk of cardiovascular diseases, and excessive generation of free radicals. Numerous lines of evidence suggested ethnomedicinal plants in order to ameliorate the disease and lessen the side effects of synthetic drugs. ${ }^{64}$ In line with this, Attia et al. ${ }^{64}$ demonstrated that $L$. sativum seeds methanolic extract, reduced blood sugar and reversed all biochemical and histological complication of alloxan-induced diabetes in rat model..$^{64}$ In another study, hypoglycaemic activity of aqueous extract of $L$. sativum seeds was examined in streptozotocin (STZ)-induced diabetic rat model. Their results displayed a significant blood glucose level reduction without any substantial alternation in basal plasma insulin concentration, which supports the concept that its hypoglycaemic activity may occur independent of insulin secretion. ${ }^{65}$ Another study performed in hypercholesterolemic albino male rats revealed that $L$. sativum seed extract improved lipid profile [decrease in cholesterol, TGs, LDL, and increase in high density lipoprotein cholesterol $(H D L)]$ and markedly diminished blood glucose in comparison to the control group. ${ }^{66}$ Furthermore, Eddouks and Maghrani67 designed a study to investigate the mechanisms underlying the hypoglycaemic activity of $L$. sativum in STZ-induced diabetic rats. Their results showed that administration of the aqueous extract decreased blood glucose, increased glycosuria, and normalized glycaemia through prevention of renal glucose reabsorption that is independent of any alternations in insulin secretion. ${ }^{67}$ L. sativum seed powder administration in alloxaninduced diabetic male Wistar rats decreased fasting blood glucose levels, glycosylated haemoglobin (Hb A1C \%), TG, lipid profile [TC and lipoprotein fractions (LDLC and VLDLC)]. The extract treatment also elevated the HDLc levels significantly. Additionally, a marked decrease in TBARS levels and increase in GSH and antioxidant enzyme activity was detected in extract treated rats. ${ }^{68}$ In a more recent study, Ullah et al. ${ }^{69}$ revealed that light (as great abiotic elicitor) play a critical role in biosynthesis of herbal metabolites. Data from their research illustrated that, callus cultures of $L$. sativum under white light exerted maximum level of phenolic profile, antidiabetic, and antioxidant properties compared to other conditions in vitro. ${ }^{69}$ It was also marked by L'hadj et al. ${ }^{70}$ that $L$. sativum flavonoid-rich extract had potential hypoglycemic, hypolipidemic, anti-inflammatory, cytoprotective, and antidiabetic properties in Wistar rats via enhancing dyslipidemia, insulin sensitivity, inflammation, and pancreas $\beta$-cell integrity. ${ }^{70}$

\section{Impacts on reproductive health}

Cumulative evidence proposed the capability of herbal medicine in improving reproductive dysfunction or fertility due to their phytochemicals. ${ }^{71}$ In a recent study in doe rabbit model, $L$. sativum oil increased the level of the reproductive hormones, while improved antioxidant status and reproductive performance (receptivity, conception rate, and litter size). ${ }^{71}$ Moreover, Kamani et al..$^{72}$ experimented efficacy of ethanolic extract of $L$. sativum seed on histopathological alternations 
of epididymis in STZ-induced diabetic adult male Wistar rats. Their findings exhibited the improved epithelium height as well as reduction of interstitial volume density, fibromuscular thickness, volume density of epithelium through preventing oxidative stress, which, in turn, demonstrated the extract's protective effect on reproductive system. ${ }^{72}$ Recently, Asl et al. ${ }^{73}$ revealed that co-administration of coenzyme Q10 (CoQ10) and $L$. sativum markedly enhanced the hypothalamic-pituitarygonadal axis activity and ameliorated the reproductive functions in adult male mice. Co-administration of CoQ10 and L. sativum resulted in elevation of all features of sexual behaviors and serum testosterone, luteinizing hormone (LH), and folliclestimulating hormone (FSH) levels as well as sperm viability and motility. ${ }^{73}$ Another animal study was performed to examine the impact of aqueous extract of $L$. sativum on fertility criteria in male mice. Findings of this study displayed that extract-treated mice had higher levels of FSH and testosterone. Overall, all the infertility parameters improved in the hyperprolactenimic animals treated with the extract. Histological analysis of the testis in the extract-treated mice exhibited normal status of seminiferous tubule with high number of sperms. ${ }^{74}$ Imade and colleagues investigated the effects of $L$. sativum seeds on the male reproductive functions in rabbit bucks. Rabbits fed with $L$. sativum seeds significantly elevated plasma LH concentrations without any significant difference in testosterone levels. Motility and live sperm percentage were significantly decreased in L. sativum seed-treated rabbits. Besides, sperm abnormality percent was increased significantly in L. sativum seed-treated rabbits dose-dependently. Taken all together, in case of high amount consumption, toxic effects of $L$. sativum seed on sperm quality and testis in rabbit bucks were observed..$^{75}$ In another study, L. sativum elevated the concentrations of estrogen, progesterone, LH, FSH, and free testosterone hormones in female rabbits. Besides, significant augmentation of sexual receptivity, conception rate, gestation length, litter size, and body weight at birth in extract treated groups were detected. Overall, L. sativum elevated reproductive hormone level and performance in vivo. ${ }^{71}$

\section{Osteoprotective activity}

Osteoporosis is a progressive "skeletal disorder characterized via low bone mass, micro-architectural deterioration of bone tissue leading to increased risk of bone fragility and fracture risk". ${ }^{53}$ In traditional medicine, $L$. sativum seeds have been proposed to have potential in healing bone fractures. ${ }^{53}$ In light of this record, recently, the fracture healing potential of the methanolic and aqueous extracts of $L$. sativum seeds in rats was experimented by Dixit et al. ${ }^{76}$ Biochemical and radiological analysis revealed that the methanolic extract markedly led to callus formation. It has been reported that ibuprofen exerted toxic effects on the osteocytes in bone tissue, whereas various concentrations of the aqueous extract of $L$. sativum seeds inhibited the effects of ibuprofen in male albino rats. ${ }^{77}$ Administration of teriparatide (a recombinant parathyroid hormone utilized as antiosteoporotic therapy) and L. sativum seeds ameliorated biochemical, histological, and morphometric bone alternations induced by glucocorticoids in male guinea pigs through osteocytes apoptosis reduction as well as osteoclasts elevation. ${ }^{78}$ Abdallah et al. ${ }^{79}$ examined osteoprotective effect of $L$. sativum extract in an ovariectomized rat model. Their findings demonstrated that the extract improved bone weight, bone formation biomarkers (LDH and osteocalcin) levels, and free radical scavenging activity (through enhancing SOD and GPX activities). Furthermore, oral administration of the extract results in increase of the bone resorption markers [e.g. carboxyterminal telopeptide, type I and, tartrate-resistant acid phosphatase (TRAP)] and regulation of receptor activator of nuclear factor kappa-B ligand/osteoprotegerin expression. Taken all together, they suggested that presence of glucosinolates, lignans, coumarins, phenolic acids, and alkaloids leads to the aforementioned antiosteoporotic effects synergistically. ${ }^{79}$ In another research, the synergistic antiosteoporotic activity of $L$. sativum and alendronate in glucocorticoid-induced osteoporosis was evaluated by methylprednisolone injection in adult female rats. Their findings revealed that $L$. sativum alone and/or in combination with alendronate treatments, markedly diminished serum TRAP and improved bone-ALP, phosphorus, calcium, and bone architecture (through increasing trabecular area or bone marrow area percentage in the proximal femoral epiphysis). ${ }^{80}$ Further, Juma ${ }^{81}$ revealed that $L$. sativum seeds significantly improved fractures healing in New Zealand white rabbits, which documented via direct measurements of callus formation in millimeters at the longitudinal medial and longitudinal lateral and circumferential areas. Administration of $L$. sativum seed powder to the rabbits with bone fractures demonstrated a significant increase in bone markers, e.g. osteopontin and vitamin D, parathormone, and lactoferrin levels as well as reduction in serum levels of osteocalcin, when compared to the untreated group. ${ }^{82}$

\section{Anti-asthmatic activity}

Bronchial asthma is a chronic inflammatory disease of airways of the lung, characterized by hyper-reactivity of the airways to various stimuli. Its clinical manifestations include paroxysmal dyspnea, wheezing cough, and a sense of thoracic constriction. From ancient times, the efficacy of the natural remedies in healing various diseases including bronchial asthma, hiccups, cough, etc. has been elucidated. ${ }^{83}$ Nevertheless, there is lack of scientific studies, which investigated the efficacy of L. sativum in bronchial asthma treatment. For that reason, Paranjape and Mehta $^{83}$ carried out a clinical trial to assess the efficiency and safety of $L$. sativum in bronchial asthma affected patients. After 4 weeks of treatment with $L$. sativum seed powder, substantial improvement in several parameters of pulmonary functions, clinical symptoms, and severity of asthmatic attacks without any adverse reaction were observed in asthmatic subjects. ${ }^{83}$ In another study carried out on guinea pigs, the bronchodilatory effect of the ethanolic extract of $L$. sativum seeds was investigated in histamine and acetylcholine induced acute bronchospasm. Data from their study showed that the extract, markedly protected guinea pigs against bronchospasm in comparison to the ketotifen and atropine sulphate (as reference drugs). ${ }^{84}$ Additionally, Rehman et al. ${ }^{85}$ indicated that a combination of anticholinergic, $\mathrm{Ca}^{2+}$ antagonist, 
and PDE inhibitory pathways were responsible for $L$. sativum's bronchodilatory activity.

\section{Neuroprotective effects}

A few studies stated potential neuroprotective activity of L. sativum. In this regard, El-Ghazouly et al. ${ }^{86}$ assessed neuroprotective effects of $L$. sativum aqueous extract on the cerebellum of adult male albino rats. Methotrexate exerted adverse effects on cerebellum by reducing the number of Purkinje cells with significant reduction of Nissl's granules. However, in methotrexate and $L$. sativum aqueous extract administrated rats approximately normal histological appearance of Purkinje cells with less vacuolated cytoplasm was observed, which was validated by a substantial rise in the Purkinje cells number, significant diminution in caspase- 3 positive cells and in GFAP immunostaining. ${ }^{86}$ Moreover, neuropharmacological impact of the alkaloid of $L$. sativum was evaluated in Swiss albino mice and Wistar albino rats. The results of this study elucidated sedative, anxiolytic, myorelaxant, and analgesic effects of $L$. sativum alkaloid through diminished locomotor activity and motor coordination, and increased preference to plus maze open arm. ${ }^{87} \mathrm{Al}$-Dbass et al..$^{88}$ examined the potential beneficial impact of $L$. sativum seed extract against glutamate excitotoxicity-induced retinal ganglion cell degeneration, which results in severe blindness. The extract enhanced the cell viability in retinal ganglion cells after exposure to the high concentrations of the glutamate. Thus, they deduced that $L$. sativum seed extracts might exert effective anti-excitotoxic and antioxidant activity in various neurological disorders. ${ }^{88}$

\section{Other medical effects}

Several studies have evaluated various beneficial effects of $L$. sativum extract in animal models. The ethanolic extract of L. sativum seeds significantly prohibited carrageenan-induced paw edema and reduced the yeast-induced hyperpyrexia in mice models and exerted anti-inflammatory and antipyretic effects, respectively. The coagulation studies demonstrated elevated levels of fibrinogen and negligible reduction in prothrombin time, which, in turn, validated the coagulant activity of the extract. ${ }^{89}$ The ethanolic extract of $L$. sativum seeds exerted significant anti-inflammatory activity in carrageenaninduced paw edema in mice through improving biomarkers of inflammation (serum albumin, C-reactive protein, and plasma fibrinogen) in comparison to the control group..$^{90}$

Alkharfy et al..$^{91}$ examined drug-herb interactions and proposed that simultaneous consumption of herbs significantly changed the phenytoin (an anticonvulsant drug) disposition in a dog model. In a follow-up study, the methanolic extract of $L$. sativum seed exerted genoprotective effect by inhibition of DNA aberrations in somatic and germ cells of mice dose-dependently. They proposed that the flavonoidal content and antioxidant activity may be responsible for this beneficial properties. ${ }^{92}$ Another in vivo study performed to evaluate the safety of $L$. sativum seeds powder in adult Wistar rats. Administration of L. sativum powder considered non-toxic and safe because of insignificant changes in food intake, gain in body weight, relative weight of organs, e.g. liver, lungs, kidney, spleen, brain, adrenals, gonads, and heart, hematological parameters [including: red blood cells (RBC), white blood cells (WBC), hemoglobin, mean corpuscular hemoglobin $(\mathrm{MCH})$, and $\mathrm{MCH}$ concentration], macroscopic and microscopic changes in vital organs, in the experimental group in comparison to the control group..$^{93}$

In a recent study, for the first time, the effects of the aqueous $L$. sativum seed extract on the immune system and general health were reported in mice model. The results demonstrated that the extract caused a boost in immune system through WBC types, RBC, and platelet counts as well as mean hemoglobin concentration, mean total body weight gains, and weights of the organs. ${ }^{94}$ Moreover, addition of $L$. sativum seeds to the diet of rats for the first 3 weeks, resulted in elevated mean body weights and body weight gains. ${ }^{95}$ In a study performed by Kaur and Sharma ${ }^{96}$, it has been elucidated that the supplementation of $L$. sativum seeds (for 2 months) moderately increased the haemoglobin $(\mathrm{g} / \mathrm{dL})$ levels among anemic adolescent girls possibly because of iron content. ${ }^{96}$

Diwakar et al. ${ }^{97}$ investigated the modulatory effect of ALA-rich $L$. sativum seed oil on lipid composition, spleen lymphocyte proliferation and inflammatory mediator production in rat model. Data from their research illustrated that the extract modulates inflammatory mediators (NO, leukotriene B4), consequently alleviates inflammatory responses. ${ }^{97}$

\section{CONCLUSION}

Various herbs and their extracts have gained substantial interest since they encompass diverse phytochemicals which represents numerous health-promoting activities. Using different parts of $L$. sativum, several pre-clinical studies demonstrated their potential in alleviating different disorders and improving health (e.g. antimicrobial, antioxidant, anticancer, antidiabetic, anti-asthmatic, and many other protective activities). Hence, L. sativum has been considered as an attractive alternative over the conventional therapeutics due to their nutritional values, and less or no adverse effects. However, further comprehensive studies are required to define molecular mechanisms underlying certain health-promoting properties and provide more convincing evidence for the efficacy of $L$. sativum.

\section{Ethics}

Peer-review: Externally peer-reviewed.

\section{Authorship Contributions}

Concept: Y.H., Y.R.S., Design: Y.H., Y.R.S., Data Collection or Processing: Y.H., T.Ö., Analysis or Interpretation: Y.H., T.Ö., Literature Search: Y.H., Y.R.S., T.Ö., Writing: Y.H., Y.R.S., T.Ö.

Conflict of Interest: No conflict of interest was declared by the authors.

Financial Disclosure: The authors declared that this study received no financial support. 


\section{REFERENCES}

1. Alshammari GM, Yahya MA, Ahmed SB. Nutritive value of elrashad (Lepidium sativum L.) seeds grown in Saudi Arabia. J Exp Biol Agric Sci. 2017;5:155-159.

2. Adam SIY, Shayma AMS, Abdelgadir WS. In vitro antimicrobial assessment of Lepidium sativum L. seeds extracts. Asian J Med Sci. 2011;3:254-261.

3. Vaishnavi RG, Choudhary P. Botanical description of garden cress (Lepidium sativum L.) plant and physical characteristics of its seeds. J Pharmacogn Phytochem. 2020;9:2424-2428.

4. Al-Snafi AE. Chemical constituents and pharmacological effects of Lepidium sativum-a review. Int J Curr Pharm Res. 2019;11:1-10.

5. Sharma S, Agarwal N. Nourishing and healing prowess of garden cress (Lepidium sativum Linn.)-a review. Indian J Nat Prod Resour. 2011;2:292297.

6. Sharma A. A comprehensive review on pharmacological properties of garden cress (Lepidium sativum) seeds. Res in Pharm Sci. 2020;10:13-18.

7. Getahun T, Sharma V, Gupta N. Chemical composition, antibacterial and antioxidant activities of oils obtained by different extraction methods from Lepidium sativum L. seeds. Ind Crops Prod. 2020;156:112876.

8. Kumar V, Tomar V, Ranade SA, Yadav HK, Srivastava M. Phytochemical, antioxidant investigations and fatty acid composition of Lepidium sativum seeds. J Environ Biol. 2020;41:59-65.

9. Rehman NU, Mehmood MH, Alkharfy KM, Gilani AH. Studies on antidiarrheal and antispasmodic activities of Lepidium sativum crude extract in rats. Phytother Res. 2012;26:136-141.

10. Agarwal J, Verna DL. Antioxidative activity and flavonoid composition from Lepidium sativum. Nat Sci. 2011;9:21-25.

11. Ghante MH, Badole SL, Bodhankar SL. Health benefits of garden cress (Lepidium sativum Linn.) seed extracts. Nuts and Seeds in Health and Disease Prevention. 2011:521-525.

12. Baregama C, Goyal A. Phytoconstituents, pharmacological activity, and medicinal use of Lepidium sativum Linn.: a review. Asian J Pharm Clin Res. 2019;12:45-50.

13. Jain T, Grover K. A comprehensive review on the nutritional and nutraceutical aspects of garden cress (Lepidium sativum Linn.). Proc Natl Acad Sci India Sect B Biol Sci. 2016;88:829-836.

14. Mohite SY, Gharal DB, Ranveer RC, Sahoo AK, Ghosh JS. Development of health drink enriched with processed garden-cress (Lepidium sativum L.) seeds. Am J Food Technol. 2012;7:571-573.

15. Sat IG, Yildirim E, Turan M, Demirbas M. Antioxidant and nutritional characteristics of garden cress (Lepidium sativum). Acta Sci Pol Hortorum Cultus. 2013;12:173-179.

16. Zia-Ul-Haq M, Ahmad S, Calani L, Mazzeo T, Del Rio D, Pellegrini N, De Feo V. Compositional study and antioxidant potential of Ipomoea hederacea Jacq. and Lepidium sativum L. seeds. Molecules. 2012;17:10306-10321.

17. Doke S, Manisha G. Garden cress (Lepidium sativum L.) seed-an important medicinal source: a review. J Nat Prod Plant Resour. 2014;4:69-80.

18. Wadhwa S, Panwar MS, Agrawal A, Saini N, Patidar LN. A review on pharmacognostical study of Lepidium sativum. ARPB. 2012;2:316323.

19. Behrouzian F, Razavi SMA, Phillips GO. Cress seed (Lepidium sativum) mucilage, an overview. Bioac Carbohydr Diet Fibre. 2014;3:17-28.
20. Ramadan MF, Oraby HF. Chapter 20-Lepidium sativum seeds: therapeutic significance and health-promoting potential. ( $2^{\text {nd }}$ eds). Nuts and Seeds in Health and Disease Prevention. 2020;273-289.

21. Kharkwal N, Prasad RV, Kumar S. Physico-chemical characterisation of Lepidium sativum (garden cress) GA-1 seed. J Pharmacogn Phytochem. 2021;10:1373-1377.

22. Shah MB, Dudhat VA, Gadhvi KV. Lepidium sativum: a potential functional food. J Ayurvedic Herb Med. 2021;7:140-149.

23. Chatoui K, Harhar H, El Kamli T, Tabyaoui M. Chemical composition and antioxidant capacity of Lepidium sativum seeds from four regions of Morocco. Evid-Based Complement Altern Med. 2020;2020:7302727.

24. El-Haggar M, El-Hosseiny L, Ghazy NM, El-Fiky FK, El-Hawiet A. Phytochemical investigation, antimicrobial and cytotoxic activities of suspension cultures of Lepidium sativum L. S Afr J Bot. 2021;138:500505.

25. Prajapati VD, Maheriya PM, Jani GK, Patil PD, Patel BN. Lepidium sativum Linn.: a current addition to the family of mucilage and its applications. Int J Biol Macromol. 2014;65:72-80.

26. Al-Otaibi MSA, Al-Quraishy S, Al-Malki ES, Abdel-Baki AS. Therapeutic potential of the methanolic extract of Lepidium sativum seeds on mice infected with Trypanosoma evansi. Saudi J Biol Sci. 2019;26:1473-1477.

27. Jasim AH. Effect of the garden cress, Lepidium sativum L. leaf extract on protoscolices of Echinococcus granulosus of sheep origin in in vitro conditions. Plant Archives. 2020;20:870-874.

28. Al-Marzoqi AH, Al-Khafaji NMS, Hussein $\mathrm{HJ}$. In vitro antibacterial activity assessment of the crude phenolic, alkaloid and terpenoid compounds extracts of Lepidium sativum $\mathrm{L}$. on human pathogenic bacteria. Int $\mathrm{J}$ Chemtech Res. 2016;9:529-532.

29. Alqahtani FY, Aleanizy FS, Mahmoud AZ, Farshori NN, Alfaraj R, Al-Sheddi ES, Alsarra IA. Chemical composition and antimicrobial, antioxidant, and anti-inflammatory activities of Lepidium sativum seed oil. Saudi J Biol Sci. 2019;26:1089-1092.

30. Berehe SG, Boru AD. Phytochemical screening and antimicrobial activities of crude extract of Lepidium sativium seeds grown in Ethiopia. Int J Pharm Sci Res. 2014;5:4182-4187.

31. Besufekad Y, Beri S, Adugnaw T, Beyene K. Antibacterial activity of Ethiopian Lepidium sativum L. against pathogenic bacteria. J Med Plant Res. 2018;12:64-68.

32. Chatoui K, Talbaoui A, Aneb M, Bakri Y, Harhar H, Tabyaoui M. Phytochemical screening, antioxidant and antibacterial activity of Lepidium sativum seeds from Morocco. J Mater Environ Sci. 2016;7:29382946.

33. Abdulrahman ZFA, Akrayi HFS, Muhammad MI. Molecular study and assessment of the medicinal potential of the Lepidium sativum plant extracts against antibiotic resistance E. coli. Med J Islamic World Acad Sci. 2012;20:98-105.

34. Omer AB, Nour AH, Ali MM, Ishag OAO, Erwa IY, Ali MA. Phytochemical screening, antimicrobial and antioxidant activity of Lepidium sativum seeds extract. SARJNP. 2020;3:10-17.

35. Stüven J, Pflugmacher S. Antioxidative stress response of Lepidium sativum due to exposure to cyanobacterial secondary metabolites. Toxicon. 2007;50:85-93.

36. Aydemir T, Becerik S. Phenolic content and antioxidant activity of different extracts from Ocimum basilicum, Apium graveolens and Lepidium sativum seeds. J Food Biochem. 2011;35:62-79. 
37. Malar J, Chairman K, Singh ARJ, Vanmathi JS, Balasubramanian A, Vasanthi K. Antioxidative activity of different parts of the plant Lepidium sativum Linn. Biotechnol Rep (Amst). 2014;3:95-98.

38. Kasabe PJ, Patil PN, Kamble DD, Dange P. Nutritional, elemental analysis and antioxidant activity of garden cress (Lepidium sativum L.) seeds. Int J Pharm Sci Rev Res. 2012;4:392-395.

39. Choudhari AS, Mandave PC, Deshpande M, Ranjekar P, Prakash O. Phytochemicals in cancer treatment: from preclinical studies to clinical practice. Front Pharmacol. 2020;10:1614. Erratum in: Front Pharmacol. 2020;11:175.

40. Bishayee A, Sethi G. Bioactive natural products in cancer prevention and therapy: progress and promise. Semin Cancer Biol. 2016;40-41:1-3.

41. Mahassni SH, Al-Reemi RM. Apoptosis and necrosis of human breast cancer cells by an aqueous extract of garden cress (Lepidium sativum) seeds. Saudi J Biol Sci. 2013;20:131-139.

42. El Sayed RA, Hanafy ZEM, Abd El Fattah HF. Possible antioxidant and anticancer effects of plant extracts from Anastatica hierochuntica, Lepidium sativum and Carica papaya against Ehrlich ascites carcinoma cells. Cancer Biology. 2020;10:1-6.

43. Selek S, Koyuncu I, Caglar HG, Bektas I, Yilmaz MA, Gonel A, Akyuz E. The evaluation of antioxidant and anticancer effects of Lepidium sativum subsp. spinescens L. methanol extract on cancer cells. Cell Mol Biol (Noisy-le-grand). 2018;64:72-80.

44. AlObaidi LAH. Study the anticancer effect of Lepidium sativum leaves extract on squamous cell carcinoma (CAL-27) cell lines. Int Res J Nat Sci. 2014;4:48-52.

45. Jahani S, Heidari Z, Azami M, Moudi B. Comparison of anticancer effects of hydroalcoholic extracts of Camellia sinensis and Lepidium sativum $L$ on HeLa cell line. Int J Cancer Manag. 2020;13.e98913.

46. Aslani E, Naghsh N, Ranjbar M. Cytotoxic effects of hydro-alcoholic extracts of cress (Lepidium sativum)-made from different stages of the plant-on k562 Leukemia cell line. HMJ. 2014;18:364.

47. Patel U, Kulkarni M, Undale V, Bhosale A. Evaluation of diuretic activity of aqueous and methanol extracts of Lepidium sativum garden cress (Cruciferae) in rats. Trop J Pharm Res. 2009;8:215.

48. Maghrani M, Zeggwagh NA, Michel JB, Eddouks M. Antihypertensive effect of Lepidium sativum L. in spontaneously hypertensive rats. J Ethnopharmacol. 2005;100:193-197.

49. Yadav YC, Srivastav DN, Seth AK, Gupta VD, Yadav KS, Sharad K. Nephroprotective and curative activity of Lepidium sativum $L$. seeds in albino rats using cisplatin induced nephrotoxicity. Pharmacologyonline. 2009;3:640-646.

50. Alsadee SAA. Hepato-nephroprotective role of Lepidium sativum against oxidative stress induced by dexamethasone in rats. Indian J Forensic Med Toxicol. 2021;15:2643-2653.

51. Nasef ANZ, Khateib BRM. Study the potential therapeutic effect of garden cress (Lepidium sativum) on nephropathy diabetic rats: biological and biochemical studies. Alex Sci Exch J. 2021;42:263-272.

52. Manohar D, Viswanatha GL, Nagesh S, Jain V, HN. S. Ethnopharmacology of Lepidium sativum Linn (Brassicaceae): a review. Int J Phytother Res. 2012;2:1-7.

53. Gilani AH, Rehman NU, Mehmood MH, Alkharfy KM. Species differences in the antidiarrheal and antispasmodic activities of Lepidium sativum and insight into underlying mechanisms. Phytother Res. 2013;27:1086-1094.
54. Najeeb-Ur-Rehman, Mehmood MH, Alkharfy KM, Gilani AH. Prokinetic and laxative activities of Lepidium sativum seed extract with species and tissue selective gut stimulatory actions. J Ethnopharmacol. 2011;134:878883.

55. Abdulmalek SA, Fessal M, El-Sayed M. Effective amelioration of hepatic inflammation and insulin response in high fat diet-fed rats via regulating AKT/mTOR signaling: role of Lepidium sativum seed extracts. J Ethnopharmacol. 2021;266:113439.

56. Al-Asmari AK, Athar MT, Al-Shahrani HM, Al-Dakheel SI, Al-Ghamdi MA. Efficacy of Lepidium sativum against carbon tetrachloride induced hepatotoxicity and determination of its bioactive compounds by GCMS. Toxicol Rep. 2015;2:1319-1326.

57. Zamzami MA, Baothman OAS, Samy F, Abo-Golayel MK. Amelioration of $\mathrm{CCl}_{4}$-induced hepatotoxicity in rabbits by Lepidium sativum seeds. EvidBased Complement Alter Med. 2019;2019:5947234.

58. Balgoon MJ. Assessment of the protective effect of Lepidium sativum against aluminum-induced liver and kidney effects in albino rat. Biomed Res Int. 2019;2019:4516730.

59. Mohamed ET, Safwat GM. Evaluation of cardioprotective activity of Lepidium sativum seed powder in albino rats treated with 5-fluorouracil. Beni-Suef Univ J Basic Appl Sci. 2016;5:208-215.

60. Shukla AK, Bigoniya P, Soni P. Hypolipidemic activity of Lepidium sativum Linn. seed in rats. J Pharm Biol Sci. 2015;10:13-22. doi:10.9790/300810441322

61. Raish M, Ahmad A, Alkharfy KM, Ahamad SR, Mohsin K, Al-Jenoobi FI, Al-Mohizea AM, Ansari MA. Hepatoprotective activity of Lepidium sativum seeds against D-galactosamine/lipopolysaccharide induced hepatotoxicity in animal model. BMC Complement Altern Med. 2016;16:501.

62. Ibrahim IA, Shalaby AA, Abdallah HM, El Zohairy NF, Bahr HI. Ameliorative effect of garden cress (Lepidium sativum L.) seeds ethanolic extract on high fat diet-prompted non-alcoholic fatty liver disease in the rat model: impact on 3-hydroxy-3-methylglutaryl-coenzyme a reductase and vascular endothelial growth facto. Adv Anim Vet Sci. 2020;8. doi:10.17582/journal.aavs/2020/8.s1.1.10

63. Sakran M, Selim Y, Zidan N. A new isoflavonoid from seeds of Lepidium sativum $L$. and its protective effect on hepatotoxicity induced by paracetamol in male rats. Molecules. 2014;19:15440-15451.

64. Attia ES, Amer AH, Hasanein MA. The hypoglycemic and antioxidant activities of garden cress (Lepidium sativum L.) seed on alloxan-induced diabetic male rats. Nat Prod Res. 2019;33:901-905.

65. Eddouks M, Maghrani M, Zeggwagh NA, Michel JB. Study of the hypoglycaemic activity of Lepidium sativum $\mathrm{L}$. aqueous extract in normal and diabetic rats. J Ethnopharmacol. 2005;97:391-395.

66. Amawi K, Aljamal A. Effect of Lepidium sativum on lipid profiles and blood glucose in rats. J Phys Pharm Adv. 2012;2:277-281.

67. Eddouks M, Maghrani M. Effect of Lepidium sativum L. on renal glucose reabsorption and urinary TGF-beta 1 levels in diabetic rats. Phytother Res. 2008;22:1-5.

68. Chauhan K, Sharma S, Agarwal N, Chauhan S, Chauhan B. A study on potential hypoglycemic and hypolipidemic effects of Lepidium Sativum (garden cress) in alloxan induced diabetic rats. Am J PharmTech Res. 2012;2:522-535.

69. Ullah MA, Tungmunnithum D, Garros L, Hano C, Abbasi BH. Monochromatic lights-induced trends in antioxidant and antidiabetic 
polyphenol accumulation in in vitro callus cultures of Lepidium sativum $\mathrm{L}$. J Photochem Photobiol B Biol. 2019;196: 111505.

70. L'hadj I, Azzi R, Lahfa F, Koceir EA, Omari N. The nutraceutical potential of Lepidium sativum $\mathrm{L}$. seed flavonoid-rich extract in managing metabolic syndrome components. J Food Biochem. 2019;43:e12725.

71. El-Speiy M, Abdella M, Abd Elaal M, Khalifah A. Effects of oral administration of Lepidium sativum, Moringa oleifera oils and aqueous extract of Vitex agnus-castus on reproductive performance and blood biochemical of doe rabbits. EJRS. 2021;31:1-24.

72. Kamani M, Hosseini ES, Kashani HH, Atlasi MA, Nikzad H. Protective effect of Lepidium sativum seed extract on histopathology and morphology of epididymis in diabetic rat model. Int J Morphol. 2017;35:603-610.

73. Asl FR, Khosravi M, Hajikhani R, Solati J, Fahimi H. Complementary effects of coenzyme Q10 and Lepidium sativum supplementation on the reproductive function of mice: an experimental study. Int $\mathrm{J}$ Reprod Biomed. 2021;19:607-618.

74. Ibraheem SR, Ibraheem MR, Hashim SS. Effect of Lepidium sativum aqueous crude extract in some fertility parameters in mice. Int $\mathrm{J} \mathrm{Sci}$ Res. 2017;6:260-266.

75. Victoria IO, Fedrick SO, Oladele G, Oluwafem AE, Atiang BJ. Effects of Lepidium sativum seed on reproductive characteristics in rabbit bucks. J Phytopharm. 2020;9:89-95.

76. Dixit Jr lii V, Kumar I, Palandurkar K, Giri R, Giri K. Lepidium sativum: bone healer in traditional medicine, an experimental validation study in rats. J Family Med Prim Care. 2020;9:812-818.

77. Abdul-Jabbar ZS, Mohammed JA. Study of histological changes in the bones of front and hind limbs of white rat treated with ibuprofen and Lepidium sativum. GEINTEC. 2021;11:1988-2003.

78. El-Haroun H, Soliman M, Soliman M, Abd El-Gawad S. Comparative study on the possible protective effect of Lepidium sativum versus teriparatide in induced osteoporosis in adult male guinea pigs. Egypt $\mathrm{J}$ Histol. 2020:931-947.

79. Abdallah HM, Farag MA, Algandaby MM, Nasrullah MZ, Abdel-Naim AB, Eid BG, Safo MK, Koshak AE, Malebari AM. Osteoprotective activity and metabolite fingerprint via UPLC/MS and GC/MS of Lepidium sativum in ovariectomized rats. Nutrients. 2020;12:2075.

80. Elshal MF, Almalki AL, Hussein HK, Khan JA. Synergistic antiosteoporotic effect of Lepidium sativum and alendronate in glucocorticoid-induced osteoporosis in Wistar rats. Afr J Tradit Complement Altern Med. 2013;10:267-73.

81. Juma Ab. The effects of Lepidium sativum seeds on fracture-induced healing in rabbits. MedGenMed. 2007;9:23.

82. Alharbi FH, Baothman OAS, Zamzami MA, Abou Gabal HH, Khoja SM, Karrouf G, Zeyadi MA, Hosawi SB, Hakeem KR, Abo-Golayel MK. Garden cress (Lepidium sativum L.) seeds enhancing osteogenesis postinducedbone fracture. Pharmacogn Mag. 2021;17:170-178.
83. Paranjape AN, Mehta AA. A study on clinical efficacy of Lepidium sativum seeds in treatment of bronchial asthma. Iran J Pharmacol Ther. 2006;5:55-59.

84. Mali RG, Mahajan SG, Mehta AA. Studies on bronchodilatory effect of Lepidium sativum against allergen induced bronchospasm in guinea pigs. Pharmacogn Mag. 2008;4:189-192.

85. Rehman NU, Khan AU, Alkharfy KM, Gilani AH. Pharmacological basis for the medicinal use of Lepidium sativum in airways disorders. Evid-Based Complement Altern Med. 2012;2012:596524.

86. El-Ghazouly DE, Mahmoud BL, Mansour MA, Konsowa EB. Histological and immunohistochemical study on the effect of methotrexate on the cerebellum of adult male albino rats and the possible protective role of Lepidium sativum. Egypt J Histol. 2021;44:241-255.

87. Shukla A, Singh R, Bigoniya P. Phytochemical and CNS activity of Lepidium sativum Linn. seeds total alkaloid. Der Pharm Lett. 2011;3:226237.

88. Al-Dbass A, Amina M, Al Musayeib NM, El-Anssary AA, Bhat RS, Fahmy R, Alhamdan MM, El-Ansary A. Lepidium sativum as candidate against excitotoxicity in retinal ganglion cells. Transl Neurosci. 2021;12:247-259.

89. Al-Yahya MA, Mossa JS, Ageel AM, Rafatullah S. Pharmacological and safety evaluation studies on Lepidium sativum $\mathrm{L}$. seeds. Phytomedicine. 1994;:155-159.

90. Yahla I, Benguiar R, Riazi A. In vivo anti-inflammatory activity of Lepidium sativum (L.) seeds. South Asian J Exp Biol. 2021;11:81-85.

91. Alkharfy KM, Al-Jenoobi Fl, Al-Mohizea AM, Al-Suwayeh SA, Khan RM, Ahmad A. Effects of Lepidium sativum, Nigella sativa and Trigonella foenum-graceum on phenytoin pharmacokinetics in beagle dogs. Phytother Res. 2013;27:1800-1804.

92. Kassem IAA, Farghaly AA, Ghaly NS, Hassan ZM, Nabil M. Composition and genoprotective effect of the flavonoidal content of Lepidium sativum L. methanolic seed extract against cyclophosphamide-induced DNA damage in mice. Pharmacogn J. 2020;12:124-130.

93. Datta PK, Diwakar BK, Viswanatha S, Murthy KN, Original report safety evaluation studies on garden cress (Lepidium sativum L.) seeds in Wistar rats. Int J Appl Res Nat Prod. 2011;4:37-43.

94. Mahassni SH, Khudauardi ER. A pilot study: the effects of an aqueous extract of Lepidium sativum seeds on levels of immune cells and body and organs weights in Mice. J Ayurvedic Herb Med. 2017;3:27-32.

95. Mahassni SH, Munshi MA. Ground Lepidium sativum Linn seeds mixed with the regular diet of rats lead to higher body weight and body weight gain. Pharmacophore. 2019;10:49-56.

96. Kaur T, Sharma M. Garden cress seeds can combat anemia. IJNAR 2016; 3:10-5.

97. Diwakar BT, Lokesh BR, Naidu KA. Modulatory effect of $\alpha$-linolenic acid-rich garden cress (Lepidium sativum L.) seed oil on inflammatory mediators in adult albino rats. Br J Nutr. 2011;106:530-539. 